Fabian Kessl

\title{
Aktivierungspädagogik statt wohlfahrtsstaatlicher Dienstleistung?
}

\author{
Das aktivierungspolitische Re-Arrangement der bundesdeutschen \\ Kinder- und Jugendhilfe
}

\begin{abstract}
Aktivierungspolitische Strategien bestimmen seit einigen Jahren die bundesrepublikanische Sozialpolitik. Eine zentrale Rolle spielt dabei die pädagogogische ReFormatierung bisheriger Vorgehensweisen: Staatliche Unterstützung wird pädagogisiert. Im vorliegenden Beitrag wird diese Entwicklung am Feld der bundesrepublikanischen Kinder- und Jugendhilfe illustriert. Damit wird deutlich, dass aktivierungspolitische Strategien keineswegs, wie oft angenommen, auf den Bereich der Arbeitsmarktpolitik und der damit verbundenen (Jugend-)Berufshilfe beschränkt bleiben. Vielmehr durchziehen aktivierungspädagogische Strategien verschiedenste sozialpolitische Felder. Betrachtet man insbesondere die Soziale Arbeit, so fällt auf, dass sie aktivierungspolitischen Strategien nicht nur ausgesetzt ist, sondern vielmehr selbst aktiv solche veränderten sozialpolitischen Maßnahmen vorantreibt. Eine kritische Soziale Arbeit als Alternative zu einer solchen „Aktivierenden Sozialen Arbeit“ müsste sich dagegen als Teil einer kritischen Sozialpolitik explizit politisch positionieren.
\end{abstract}

\section{Einleitung}

Im Kapitel „Arbeitsmarkt“ des Ende 2005 unterzeichneten Koalitionsvertrag von CDU, CSU und SPD ist folgender Hinweis zu lesen: „Das neue System der Grundsicherung für Arbeitsuchende sieht eine konsequente Aktivierung insbesondere junger hilfebedürftiger erwerbsfähiger Menschen vor“. Auf der Homepage des Bundesministeriums für Familie, Senioren, Frauen und Jugend findet sich in der Rubrik „Kinder und Jugend“ seit Anfang Februar 2006 der Themenschwerpunkt „Aktivierung von Potenzialen. Jugend gestaltet das Land“. Dort heißt es: „Kinder und Jugendliche wollen und sollen in die politischen Entscheidungs- und Gestaltungsprozesse einbezogen werden; sie wollen und sollen ihre Bedürfnisse und Interessen einbringen und vertreten können“. ${ }^{1}$

Diese beiden Passagen stehen exemplarisch für eine Vielzahl von politisch-programmatischen Texten, in denen die Aktivierung junger Menschen in den letzten Jahren zu einem sozialpolitischen Schlüsselthema gemacht wurde (vgl. Beiträge in Dahme et al. 2003). Das Modell des „Aktivierenden Staats“ und eine damit verbundene ,ak-

Siehe $\quad$ http://www.bmfsfj.de/Politikbereiche/kinder-und-jugend, did=67068. html (Stand: 3. Mai 2006). 
tivierende Sozialpolitik“ sind zu den programmatischen Leitbildern „modernen Regierens" erklärt worden. Aktivierung wird dabei als Mobilisierung subjektiver Lebensgestaltungspotenziale und einer damit verbundenen Lebensgestaltungsverantwortung verstanden. Entsprechende Aktivierungsmaßnahmen werden primär auf die Gesellschaftsmitglieder ausgerichtet, denen ein unzulässiges Maß an Passivität unterstellt wird. Dabei wird vor allem aus zwei Richtungen argumentiert. Politisch-rhetorisch und medial wird die Figur des ,faulen Arbeitslosen“ und des "Sozialschmarotzers" skandalisiert. Diskursrelevant waren für die jüngste aktivierungspolitische Debatte eine entsprechende Behauptung des ehemaligen Bundeskanzlers Gerhard Schröder im Frühjahr 2001 und die seit 2003 inszenierten Missbrauchsgeschichten, die vor allem die Bildzeitung dynamisiert hat. Ihren Anfang nahmen diemedialen Dramatisierungen mit dem Bericht über den angeblichen Sozialhilfebetrüger „Florida-Rolf“ ${ }^{2}$ Gleichzeitig wird von Seiten der sozialwissenschaftlichen Politikberatung ein struktureller Grund für diese Passivität identifiziert. Denn die bisherigen staatlichen Sozialleistungen selbst seien der Grund für die Erwerbslosigkeit (vgl. Sinn et al. 2002: 8-10). Denn die garantierten Sozialhilfeleistungen führten dazu, dass erwerbslose Gesellschaftsmitglieder keinen Grund hätten, eine gering bezahlte Beschäftigung zu übernehmen, obwohl genau in diesem Segment der so genannten „Niedriglohnjobs“ das größte Arbeitsplatzpotenzial bereit liege. Vor dem Hintergrund einer solchen Annahme kommen Aktivierungsprotagonisten á la Hans-Werner Sinn dann zu der bemerkenswerten Einschätzung, dass die Menschen, ,die von diesem Problem betroffen sind, das Recht auf Arbeit faktisch verwehrt (wird)“ (ebd.: 9). Wohlfahrtsstaatliche Unterstützungsleistungen werden somit als arbeitsmarktpolitisch kontraproduktive Maßnahmen redefiniert. Für die Soziale Arbeit und die Kinder- und Jugendhilfe wird gefolgert, dass die bisherige sozialpädagogische Handlungsmaxime der Unterstützungsorientierung grundlegend überdacht werden müsse. Es müsse nun eine neue Formel gelten, nämlich „Handlungsdruck statt Übernahmegarantie“ (Esch et al. 2001: 522). Dementsprechend stehen die Arbeitsmarktpolitik und im sozialpädagogischen Bereich die Berufshilfe bzw. Beschäftigungs- und Beschäftigungsvorbereitungsmaßnahmen im Zentrum der neuen Aktivierungspolitiken, wie das Eingangszitat aus dem Koalitionsvertrag verdeutlicht. Auch die wissenschaftlichen Auseinandersetzungen beziehen sich daher bisher primär auf diesen Bereich bundesrepublikanischer Sozialpolitik. Demgegenüber soll im weiteren Text am Beispiel der bundesrepublikanischen Kinder- und Jugendhilfe aufgezeigt werden, dass Aktivierungsstrategien keineswegs auf diesen Bereich beschränkt bleiben, wie das zweite Eingangszitat von der Ministeriumshomepage auch bereits andeutet. Damit rückt ein zweiter Aspekt der jüngsten aktivierungspolitischen Strategien ins Zentrum, der zwar gelegentlich erwähnt (vgl. Ehrke 1999; Evers 2000: 27), dessen Bedeutung für die angestrebte „Aktivierende Sozialpolitik“ aber

Debatten über einen angeblichen Missbrauch von Sozialleistungen begleiten die politischen Diskussionen um mögliche Reaktionsmuster auf das Mitte der 1970er Jahre erstmals diagnostizierte Phänomen einer „Massenarbeitslosigkeit“ regelmäßig (vgl. Oschmiansky 2003). 
noch nicht hinreichend wahrgenommen wurde: Die aktivierungspolitisch angestrebten Vorgehensweisen sind pädagogischer Natur. Zentrales Prinzip der aktivierungspolitischen Strategien der letzten Jahre ist daher auch eine staatlich inszenierte $A k$ tivierungspädagogik. Darauf weisen eine wachsende Zahl von erziehungswissenschaftlichen Studien und dort vor allem Arbeiten aus dem Bereich der Sozialen Arbeit inzwischen hin (vgl. Kessl 2005; Sturzenhecker 2003; Winkler 2006: 217-222; Ziegler 2004).

\section{Aktivierungspolitik - zum Stand der wissenschaftlichen Reflexion}

Drei idealtypisch differenzierbare Positionen prägen die wissenschaftlichen Auseinandersetzungen um die jüngsten aktivierungspolitischen Strategien in den Feldern der Sozialpolitikforschung.

(1) Affirmative Beiträge zur Frage einer neuen Staatlichkeit unter dem Leitbild „Aktivierung“. Mit Verweis auf ein angebliches Leistungsversagen, immense Finanzierungprobleme und scheinbar konstitutive Gerechtigkeitsdefizite (Lessenich/Möhring-Hesse 2005: 91-115) werden seit Ende der 1990er Jahre verstärkt sozialpolitische Neujustierungen gefordert, die dem bisherigen Interventionsstaat Ineffizienz und Ineffektivität bescheinigen und für den Aufbau eines Gewährleistungsstaats plädieren (vgl. Bandemer/Hilbert 1998). Aktivierung wird in solchen Konzeptionen einer „Aktivierenden Sozialpolitik“ zum zentralen Handlungsprinzip erklärt. Besonders einflussreich sind diese Beiträge nach dem Regierungswechsel 1998 im Umfeld der bundesrepublikanischen Sozialdemokratie geworden (Streeck/Heinze 2000, Hombach 1998). So schrieb der damalige Wahlkampfmanager des späteren Bundeskanzler Schröder in seiner Programmschrift zur Politik der neuen Mittte: Staatliche Maßnahmen dürften sich „eben nicht mehr in Verteilung erschöpfen, sondern [sollten] positiv der Befähigung, der Aktivierung zur Selbstverantwortung dienen“ (Hombach 1998: 70). Mit dem Regierungswechsel 2005 wurde die Semantik der Aktivierung zwar deutlich reduziert, um dem Eindruck entgegen zu wirken, die Politik der rot-grünen Vorgängerregierung würde nur verstetigt. So findet sich beispielsweise in der Koalitionserklärung der so genannten Großen Koalition nur ein einziges Mal das Wort „Aktivierung". Zugleich werden die aktivierungspolitischen Programme keineswegs von der Agenda genommen, wie die eingangs zitierte Textstelle aus dem Koalitionsvertrag bereits verdeutlicht. Semantisch kaschiert ist zwar nun die Rede von einer notwendigen „größeren Eigenverantwortung“, die die und der Einzelne zu übernehmen habe und für die ein „Bewusstsein in der Bevölkerung“ herzustellen sei. Die bereits beschlossenen und noch vorgesehenen Verschärfungen im Bereich der Arbeitsmarktpolitik und Berufshilfe sind aber Beleg dafür, dass die unter RotGrün implementierte Aktivierungspolitik nicht nur weitergeführt, sondern noch verschärft werden soll (vgl. „Änderungsgesetz“ und „Optimierungsgesetz“ zum SGB II 2006). Zur öffentlichen Legitimation aktivierungspolitischer Vorgehensweisen tragen einflussreiche (neo)liberale und (neo)konservative Zeitdiagnostiker bei, die darauf verweisen, dass nur eine Ablösung des bisherigen Prinzips der Ver- 
teilungsgerechtigkeit zugunsten einer „Chancengerechtigkeit“ die aufgebauten gesellschaftlichen Blockaden niederreißen könne (Nolte 2006: 226). Linke wie rechte Politikstrategien müssten endlich darauf abgestellt werden, so die Behauptung, die ,Selbstverantwortung“ der einzelnen Gesellschaftsmitglieder zu aktivieren (ebd.: 237; vgl. di Fabio 2005). Die bisher angestrebten egalitären Programme der Verteilungsgerechtigkeit hätten nämlich zur Blockade von Entwicklungspotenzialen und damit zur Benachteiligung des „Standorts Deutschland“ geführt. Nicht mehr die Gleichheit der Lebenslagen und der Bedingungen individueller Lebensführung könne das Ziel staatlicher Maßnahmen darstellen, sondern die Bereitstellung gleicher Chancen für die unterschiedlichen individuellen Beteiligungs- und Entwicklungsmöglichkeiten. Nicht soziale Ungleichheit sei das Problem, sondern die Möglichkeiten der Entfaltung vorhandener Potenziale.

Gerechtigkeitstheoretisch setzen die Aktivierungspropheten zur Legitimation entsprechender politischer Strategien somit einseitig auf die Freiheitsdimension menschlichen Handelns. Diese gelte es gegenüber der Gleichheitsdimension zu maximieren. Demgegenüber ist der zentrale Ansatzpunkt für die zweite - kritische - Position hinsichtlich der aktivierungspolitischen Strategien im Feld der Sozialpolitikforschung gerade die Überzeugung, dass individuelle Freiheitsrechte und die Sicherung eines bestimmten Maßes an universeller Gleichheit im Sinne einer Garantie sozialer Teilhabe nur zusammen denkbar seien.

(2) Sozial- und gerechtigkeitstheoretische Einwände gegen die Reduzierung sozialer Rechte. Die spezifische neoliberale Prägung der aktuell vorherrschenden aktivierungspolitischen Programmatiken sei offensichtlich, so wenden die Kritiker der aktuellen aktivierungspolitischen Strategien ein (vgl. Mahnkopf 2000). Denn der Blick in fundamentale Sozialstaatskritiken, wie sie von neoliberalen Denkern á la Hayek schon vor 50 Jahren geäußert wurden, mache deutlich, wes Geistes Kind solche Forderungen nach Chancengerechtigkeit seien: „Der Grund dafür, daß viele der neuen Wohlfahrtsmaßnahmen der Regierung eine Bedrohung der Freiheit darstellen, ist, daß sie, obwohl sie als bloße Dienstleistungen dargestellt werden, in Wirklichkeit eine Ausübung der Zwangsgewalt der Regierung darstellen“ (Hayek 1991[1960]: 329; vgl. aktuell dazu u.a. die Erklärungen im Umfeld der arbeitgeberfinanzierten „Initiative Neue Soziale Marktwirtschaft“, vgl. http://www.insm.de, Stand: 17. Februar 2006). Soziale Ungleichheit werde vom angestrebten „Sozialinvestitionsstaat“ akzeptiert, da dieser nicht mehr dazu verpflichtet werden soll, menschliche Notlagen zu substituieren, das heißt zumindest ein gewisses Maß an Gleichheit herzustellen, sondern im Vorfeld das „Humankapital“ der Gesellschaftsmitglieder möglichst effektiv und effizient zu aktivieren. Gegen die damit verbundene Überzeugung, dass das Setzen auf eine solche „Beschäftigungs- und Eigenverantwortungsbefähigung" notwendigerweise ein staatliches Freisetzungsprogramm darstellen müsse (vgl. Gerdes 2006: 15), wenden kritische Positionen ein, damit würden die ungleich verteilten Ausgangsbedingungen ignoriert.

Die Implementierung der so genannten Hartz-Gesetze hat bereits vor dem InKraft-Treten des „Vierten Gesetzes für moderne Dienstleistungen am Arbeitsmarkt" (Hartz IV) zu weiteren vehementen Einwänden gegenüber den politischen Aktivierungsstrategien geführt (vgl. Eick/Sambale 2005; Dahme/Wohlfahrt 2005; Gillen 2004). Sozialtheoretisch wird sowohl auf einen damit verbundenen Paradig- 
menwechsel vom bisherigen wohlfahrtsstaatlichen hin zu einem sicherheitsstaatlichen und workfare-basierten Arrangement aufmerksam gemacht (vgl. Beiträge in Widerspruch 46/2004; Freyberg 2003; Maaser 2006; Wölfle/Schöller 2004) als auch auf eine Redefinition bestehender (teil)kollektiver Sicherungsstrukturen im Fall alltäglicher Lebensrisiken (Krankheit, Unfall, Erwerbslosigkeit) (vgl. Castel 2005). Während in den sozialversicherungsrechtlichen Teilgemeinschaften der Risikoungleichen Lebensrisiken im wohlfahrtsstaatlichen Arrangement wenigstens teilweise substituiert wurden, werden stattdessen Versicherungsgemeinschaften Risikogleicher auf- und ausgebaut (vgl. Schmidt-Semisch 2002).

(3) Vermittelnde Position. Eine dritte Gruppe von Sozialpolitikforschern versucht eine vermittelnde Position zwischen diesen beiden Polen auszuloten (vgl. Olk 2000; Evers 2000). Unter der Überschrift der „Ermöglichung“, mit der Anschluss genommen wird an die englischsprachigen Enabling-Konzepte (vgl. Gilbert/Gilbert 1989), wird einerseits die Privatisierung von Risiken der Lebensführung zurückgewiesen und für eine Beibehaltung wohlfahrtstaatlicher Sicherungsstrukturen argumentiert. Andererseits wird es auch als notwendig erachtet, die Verquickung von Verpflichtungen und sozialen Rechten zu betonen: Universelle Anspruchsberechtigungen sollten mit einem „Paket an ,ermöglichenden“ und aktivierenden Maßnahmen verbunden werden“, so formuliert beispielsweise Thomas Olk (2000: 117).

Neben diesen theorie-konzeptionellen Positionen findet sich inzwischen auch eine wachsende Zahl von empirischen Studien, in denen untersucht wird, welche Form die Umsetzung der Aktivierungsstrategien annimmt (vgl. Dingeldey 2003, 2005; Walther 2003). Diese Studien konzentrieren sich bisher vor allem darauf, pfadabhängige Gestaltungsdifferenzen in den verschiedenen nationalstaatlichen Aktivierungsprogrammen und dort wiederum in den unterschiedlichen sozialpolitischen Handlungsfeldern nachzuzeichnen. Demgegenüber verweisen die bereits genannten Arbeiten im Feld Sozialer Arbeit - vor allem aus einer machtanalytischen und feldtheoretischen Perspektive - in jüngster Zeit verstärkt auf Regelmäßigkeiten dieser veränderten politischen Rationalitäten über die Grenzen der einzelnen Handlungsfelder und Welfare Regimes hinweg (vgl. auch die Beiträge in Marston/McDonald 2006).

In diesen Arbeiten wird vor allem auf eine bestimmte Form der Thematisierung hingewiesen: Mit der Implementierung des Aktivierungsprinzips in einer wachsenden Zahl sozialpolitischer Handlungsfelder (Arbeitsmarkt, Gesundheitsversorgung, Alterssicherung, Kinder- und Jugendhilfe) und verschiedenen Welfare Regimen erfahre Aktivierungspädagogik in einer bestimmten Form ein beachtliches Comeback bzw. werde in neuer Weise rekonstruiert. Oder etwas zugespitzt formuliert: Staatliche Instanzen behandelten die Gesellschaftsmitglieder zunehmend so als hätten sie es nicht mehr mit „(potenziellen) Erwachsenen“, sondern mit „,(potenziellen) Kindern“ zu tun (Sturzenhecker 2003: 389-390). Nicht zufällig kommt ein größerer Anteil dieser Arbeiten aus dem Feld der erziehungswissenschaftlichen Forschung und hier vor allem aus dem Bereich der Sozialen Arbeit. Denn in den entsprechenden (sozial)pädagogischen Arbeitsfeldern sind aktivierungspädagogische Strategien zum einen prinzipiell nichts Neues, und zugleich sieht sich gerade die Soziale Arbeit in der Jugendberufshilfe, aber eben 
auch in der Schulsozialarbeit, in stadtteilbezogenen Arbeitsansätzen, den Hilfen zur Erziehung oder in der Kinder- und Jugendarbeit mit den aktuellen aktivierungspolitischen Forderungen besonders deutlich konfrontiert. Diese Forderung wird fälschlicherweise zumeist als Forderung „,von außen“ betrachtet, obwohl die weiter unten vorgenommene dispositivanalytische Rekonstruktion dominierender politischer Rationalitäten zeigt, dass Fachkräfte und Träger der Kinder- und Jugendhilfe aktivierungspolitische Strategien selbst an vielen Stellen vehement mit befördern (vgl. Kessl 2006).

\section{Aktivierung - das (sozial)pädagogische Prinzip}

Aktivierung kann als das pädagogische Prinzip an sich bezeichnet werden (vgl. Kessl/Otto 2003). Denn Ziel pädagogischer Maßnahmen ist seit den Tagen der Aufklärung, den Einzelnen durch ein bestimmtes Maß an Fremdführung zur subjektiven Selbstführung zu bringen.

Das Subjekt „lernt, über sich selbst zu verfügen, seine eigenen Perspektiven $\mathrm{zu}$ fassen und $\mathrm{zu}$ verfolgen; es wird imstande, sich selbst $\mathrm{zu}$ erziehen", so beschreibt beispielsweise Michael Winkler am Ende seiner Theorie der Sozialpädagogik das Ergebnis einer gelungenen (sozial)pädagogischen Intervention (Winkler 1988: 335). Während Platon im Höhlengleichnis noch eine gewaltsame Herausführung des unkundigen Menschen aus der fast dunklen Höhle in das Sonnenlicht der Erkenntnis fordert und dafür nur eine kleine Zahl von Auserwählten für fähig hält, wird der Mensch von den Aufklärern und damit den Vordenkern einer „modernen“ Pädagogik zum selbstbestimmten Gehen aufgefordert. Das eigene Gehen scheint allerdings auch Rousseau wie Kant nur unter Führung anderer erlernbar. Die noch ungebildeten Menschen erscheinen in diesem Sinne nicht gehfähiger als die Knaben im antiken Athen. Die notwendige Fremdführung zur späteren (mündigen) Selbstführung bleibt das Credo pädagogischer Handlungskonzeptionen. Nicht einmal die gegenüber jeglichen pädagogischen Interventionen höchst skeptischen Reform- und Anti-Pädagogen des 20. Jahrhunderts haben dies jemals bestritten.

Das Ziel pädagogischer Intervention scheint daher unzweifelhaft, streitbar allerdings der Weg dorthin. In welcher Form zu welchem Ziel ,,aktiviert“" wird, das ist der eigentliche pädagogische und erziehungswissenschaftliche Streitpunkt. Für die Soziale Arbeit und die Kinder- und Jugendhilfe lässt sich diese Erkenntnis etwa folgendermaßen zusammenfassen: Das Prinzip der „Hilfe zur Selbsthilfe“ wird von der absoluten Mehrheit geteilt. Die Frage ist nur, welche Form diese Hilfe annehmen soll und wann die Hilfe als erfolgreich verbucht werden kann. Diese Frage der Gestaltung (sozial)pädagogischer Interventionen ist immer eine Frage in Bezug auf (sozial)politisch vorherrschende Vereinbarungen zur Gestaltung des Sozialen. Dementsprechend problematisieren sozialpädagogische Denker und Fachkräfte häufig und seit Beginn der Institutionalisierung Sozialer Arbeit den sozialpolitischen Kontext hinsichtlich seiner sozialpädagogischen Implikationen. Petersen eröffnete beispielsweise 1976, also im Kontext der ersten Massenarbeitslosigkeitsdebatten, den 68. Deutschen Fürsorgetag, der bereits unter dem Titel 
„Selbsthilfe und ihre Aktivierung durch die Soziale Arbeit“ stand, mit folgenden Worten:

Zum Thema des Fürsorgetages darf ich bemerken: Der Ausbau des Systems der sozialen Sicherheit und das Streben nach mehr sozialer Gerechtigkeit haben als unausweichliche Begleiterscheinung mit sich gebracht, daß die Abhängigkeit des einzelnen von der Vorsorge und Fürsorge des Staates in den letzten Jahrzehnten größer geworden ist. Diese Entwicklung macht es erforderlich, die Inhalte und Wirkungen der sozialen Leistungen ständig kritisch daraufhin zu überprüfen, ob sie geeignet sind, den Bürger in seiner Eigenpersönlichkeit zu stützen und zu stärken. Außerdem gibt es eine Anzahl von Bürgern, die Hilfen zur Aktivierung ihrer Selbsthilfekräfte benötigen (Petersen 1976: 2).

Aktivierung als Konsequenz einer sozialstaatskritischen Analyse ist also keineswegs erst mit den neokonservativen und neoliberalen Zeitdiagnosen á la Nolte auf die Agenda gesetzt worden. Ganz im Gegenteil: Sozialpädagogische Programme sind seit ihrer Implementierung als Teil des wohlfahrtsstaatlichen Arrangements von einem markanten anti-staatlichen Impuls geprägt, der sich aus dem Verdacht speist, die angestrebte, das heißt zu aktivierende subjektive Selbstführung müsse an der staatlichen Leine scheitern, an die die betroffenen Gesellschaftsmitglieder in staatlichen Erziehungs- und Bildungsinstitutionen gelegt werden. Doch nicht nur das. Das soziale Integrationsversprechen des Wohlfahrtsstaats wurde als selbstverständlich vorausgesetzt, obwohl die anti-staatliche Grundhaltung hier hätte skeptisch stimmen müssen. Doch damit wäre die Einbindung in die institutionalisierte Form des wohlfahrtsstaatlichen Arrangements zu thematisieren gewesen und diese garantiert nun zugleich die Etablierung Sozialer Arbeit als sozialer Dienstleistung im 20. Jahrhundert. Durchaus analog zu strukturfunktionalistischen Argumentationen wurde davon ausgegangen, dass das wohlfahrtsstaatliche Arrangement als eine Zusammensetzung unterschiedlicher Interventionsformen betrachtet werden müsse: rechtliche, ökologische, ökonomische und eben auch pädagogische (vgl. Kaufmann 2002). Die pädagogische Intervention zielt in dieser Deutung primär auf Formen der Lebensführung (Kompetenzen) - nicht zuletzt durch die für alle Kinder und Jugendlichen verpflichtende schulische Sozialisation. Die damit auch markierte konstitutive Staatlichkeit Sozialer Arbeit als Teil des wohlfahrtsstaatlichen Arrangements (vgl. Schaarschuch 2003) wird allerdings in den Diskussionen Sozialer Arbeit oft ausgeblendet. Damit geraten Soziale Arbeit und Kinder und Jugendhilfe aber in die Gefahr, ihre konstitutive Rolle als (Re)Produzentin vorherrschender Rationalitäten zu übersehen - eine in Zeiten aktivierungspädagogischer Konjunkturen politisch besonders leichtsinnige Haltung. Denn die jüngsten aktivierungspolitischen Strategien werden als (sozial)politischer Übergriff in die Felder Sozialer Arbeit betrachtet, und die eigene (Re-)Produktionsrolle als Teil des wohlfahrtsstaatlichen Arrangements und damit eben auch als Teil der aktuell dominierenden sozialpolitischen Strategien im Aktivierungsgewand gerät aus dem Blick.

Fasst man demgegenüber Soziale Arbeit als konstitutiven Teil des wohlfahrtsstaatlichen Arrangements, lässt sie sich als Bestandteil von dessen pädagogischer Institutionalisierung fassen. Pädagogische Intervention kann dann als aktive Unter- 
stützung und geplante Beeinflussung von Lebensführungsweisen definiert werden (vgl. Kessl 2005: 35-66). Sozialpädagogische Maßnahmen werden im wohlfahrtsstaatlichen Arrangement somit als solche pädagogische Maßnahmen bestimmt, die im Fall menschlicher Notlagen als notwendig angesehen werden. Das heißt sozialpädagogische Maßnahmen sollen dann greifen, wenn die Lebensführung von einzelnen Gesellschaftsmitgliedern aufgrund sozialer Risiken derart beeinträchtigt ist, dass diese nicht mehr relativ selbstbestimmt möglich ist. Kinder- und Jugendhilfe stellt schließlich den Teilbereich Sozialer Arbeit dar, in dem sozialpädagogische Maßnahmen mit Blick auf die nachwachsende Generation realisiert werden. Soziale Arbeit und Kinder- und Jugendhilfe sind damit aber auch nicht auf den Bereich pädagogischer Interventionsformen im Kaufmannschen Sinne zu beschränken. Soziale Arbeit stellt vielmehr eine Teilform der Sozialpolitik dar. Ihr Ansatzpunkt ist zwar ein pädagogischer, insofern sie primär und zuvorderst auf die Disposition und nicht die Position der Betroffenen zielt (vgl. Ziegler 2004: 83-84). Insgesamt ist sie aber, so könnte man in kritischem Anschluss an Kaufmanns Modell formulieren, auch Konsequenz und Ausdruck rechtlicher Ansprüche, Teil der infrastrukturellen Angebote im wohlfahrtsstaatlichen Arrangement, und Vermittlungsinstanz zu Geldleistungen. Während Soziale Arbeit im Kontext des bisherigen wohlfahrtsstaatlichen Arrangements also keineswegs auf rein pädagogische Leistungen beschränkt war, waren pädagogische Vorgehensweisen im Feld des Sozialen aber weitgehend auf die Arbeitsbereiche Sozialer Arbeit beschränkt. Das heißt wohlfahrtsstaatliche Leistungen, wie beispielsweise das Arbeitslosengeld wurden systematisch von fürsorgerischen Leistungen unterschieden, wie sie in der Kinder- und Jugendhilfe (z.B. der Jugendsozialarbeit) anzutreffen waren.

Demgegenüber werden im Rahmen der aktuellen aktivierungspolitischen Neujustierung des Sozialen - man kann insofern von einer neo-sozialen Transformation des wohlfahrtsstaatlichen Arrangements sprechen (vgl. Kessl/Otto 2003; Lessenich 2003; Richter 2004; Ziegler 2004) - staatliche Maßnahmen selbst pädagogisiert.

\section{Subjektive Lebensgestaltungsverantwortung - die aktivierungs- pädagogische Rethematisierung der Kinder- und Jugendhilfe}

Im Folgenden soll dargestellt werden, wie sich der Diskurs der bundesdeutschen Kinder- und Jugendhilfe angesichts der aktivierungspolitischen Neujustierung des Sozialen verändert hat. Hierzu wird in den Blick genommen, wie sich die Transformation des politischen Steuerungsmodus im konkreten sozialpädagogischen Handlungsvollzug widerspiegelt. Dazu bietet sich die Inblicknahme einer spezifisch bundesdeutschen Institution an: der Landesjugendämter. ${ }^{3}$ Denn die Landes-

Die föderale Einrichtung von Landesjugendämtern ist seit Ende der 1990er Jahren in einigen Bundesländern verstärkt in die Kritik geraten, beispielsweise in Hessen. Landesjugendämter waren allerdings bereits vor Beginn ihrer ersten gesetzlichen Konstitution umstrittene Einrichtungen. Christa Hasenclever (1978: 71) weist in ihrer Historiographie der Jugendhilfe und der Jugendgesetzgebung auf den kommunalen Widerstand gegen deren Einrichtung Anfang der 1920er Jahre hin. Kommunen sa- 
jugendämter bilden einen Transformationsriemen zwischen den Landesministerien als politischer Steuerungsinstanz und den Kreis- und Stadtjugendämtern als verantwortlicher Steuerungsinstanz für die Gestaltung, Genehmigung und Kontrolle der einzelnen sozialpädagogischen Maßnahmen vor Ort. Die Aufgaben der Landesjugendämter beschränken sich dementsprechend vor allem auf Fortbildungs-, Beratungs- und Informationstätigkeiten. In diesem Zusammenhang publizieren fast alle Landesjugendämter regelmäßig ein Periodikum. Das dem weiteren Text zugrunde liegende Material ist Teil einer dispositivanalytischen Studie, die auf Basis zweier Jahrgänge aller bundesrepublikanischen Landesjugendamts-Periodika erstellt wurde (vgl. Kessl 2005: 123-211).

Eine erste zentrale aktivierungspädagogische Thematisierungslinie ist die Rede von der Prävention. Im thüringischen Landesjugendamt-Info wird über einen suchtpräventiven Wettbewerb für thüringische Schüler berichtet. Diese werden in diesem Wettbewerb unter dem Titel „Be smart - don't start“ aufgefordert, ein halbes Jahr als „Nichtraucherklasse“ aufzutreten (Th 200: 49). ${ }^{4}$ Zur Teilnahme motivieren soll die Schüler, die kalkulative Einsicht in die „Kosten“ des Rauchens: die ,persönliche“ Gesundheit, „subjektive“ Lebensgestaltungsmöglichkeiten und „,individuelle“ Lebensqualität - unter Umständen sogar das „eigene“ Leben. ${ }^{5}$ Dass eine gemeinschaftliche Entscheidung zum Nichtrauchen kalkulativ erfolgreicher sei als eine individuelle Entscheidung zum Rauchen, soll den Schülern dadurch verdeutlicht werden, dass der Siegerklasse eine gemeinsame Klassenfahrt als Preis winkt. Und damit wird bereits eine zweite Thematisierungslinie deutlich: die Rede von der zweckrationalen Kalkulation. Kinder und Jugendliche sollen ihr Handeln und damit das persönliche Risiko möglichst zielgenau kalkulieren. Nun ist das thüringische Beispiel nicht zufällig aus dem Bereich der Schule und damit eigentlich nicht aus dem direkten und typischen institutionellen Kontext der Kinder- und Jugendhilfe - wenn auch Kinder- und Jugendhilfe zunehmend schulbezogen tätig wird (Schulsozialarbeit, Ganztagsschulangebote, Elternarbeit). Denn die Kinder und Jugendlichen, die Angebote der Kinder- und Jugendhilfe in Anspruch nehmen oder aufgrund einer Elternentscheidung oder eines Familiengerichtsbeschlusses in Anspruch nehmen müssen, sind nun bereits ,,in den Brunnen gefallen“, so kann man in

hen sich damals in ihrer Selbstverwaltung begrenzt und befürchteten einen ihres Erachtens unnötigen Kostenaufwand. Diese Einwände konnten sich im Einführungsgesetz zum RJWG 1924 insofern auch durchsetzen, als nach Artikel 1 der „Verordnung über das Inkrafttreten des Reichsgesetzes für Jugendwohlfahrt" die Errichtung von LJÄn dem Ermessen der Länder anheim gestellt wurde. Der entsprechende $§ 12$ des RJWG wurde als $\S 19$ des JWG 1961 wieder in Kraft gesetzt. Eine strukturanaloge Argumentation führte knapp 80 Jahre später zur Auflösung des hessischen Landesjugendamtes.

Die Signaturen verweisen auf die jeweiligen Fundstellen im untersuchten Material: Herausgeberin, das heißt das jeweilige Landesjugendamt (z.B. „Th“ für Thüringisches Landesjugendamt), Erscheinungszeitpunkt, das heißt Heftnummer und Jahreszahl (z.B. „200“ für Heft 2 im Jahr 2000) und Textstelle, das heißt Seitenzahl bzw. Seitenzahl plus Spaltenzahl (z.B. „4,3“ für Seite 4, Spalte 3) (vgl. ausführlich Kessl 2005: 131-211).

Dementsprechend lautet auch eine der Formeln, mit denen innerhalb der Europäischen Union auf Zigaretten- und Zigarrenpackungen auf die Gefahr des Rauchens aufmerksam gemacht werden soll: „Rauchen kann tödlich sein“. 
die Präventionssemantik formulieren. Dementsprechend wird diesen Kindern und Jugendlichen auch zunehmend unterstellt, sie hätten zumindest ein bestimmtes Maß an „Risikokalkulation“ bereits vermissen lassen, wären ihrer subjektiven Lebensgestaltungsverantwortung - eine weitere Thematisierungslinie - also nicht ausreichend nachgekommen. Daher werden für sie immer häufiger Maßnahmen vorgehalten, wie sie beispielsweise im Landesjugendamt-Info des Landschaftsverbandes Württemberg-Hohenzollern beschrieben werden. Hier findet sich ein Bericht über ein BoxTraining für Jugendliche. Bei diesem ,sozialen Trainingskurs“ gehe es neben dem Training der korrekten Boxtechnik daher vor allem um Fähigkeiten, wie ,sich Einund Unterordnen können, Durchhaltevermögen, Disziplin und [...], einstecken zu können ohne auszurasten“ (WH 100: 20). Dazu müsse das „Training [...] minutiös durchorganisiert“ und nach ,verbindlichen Regeln“ aufgebaut werden, deren strikte Einhaltung eine Teilnahmevoraussetzung darstelle (WH 100: 20-21): „Pünktliches Erscheinen in Sportkleidung, Teilnahmepflicht an allen Bestandteilen des Trainings sowie beim Auf- und Abbau der Geräte, Stehen bleiben beim zugewiesenen Gerät bis zum nächsten Trainingsintervall und Aufmerksamkeit und Schweigen, während der Trainer spricht“. Und ,wer wiederholt gegen die Regeln verstößt [...] fliegt raus“" (WH 100: 21).

Diese Verregelung sozialpädagogischer Settings ist Teil eines Perspektivwechsels durch Verhaltenstrainings statt sozialpädagogischer Lern- und Bildungsangebote. Denn, so ist im rheinland-pfälzischen Landesjugendamts-Info zu lesen, „,[n]ur eine intensive pädagogische Arbeit könnte wieder ,herrichten“, was an ihnen [den Jugendlichen, F.K.] in ihrer bisherigen Entwicklung versäumt worden sei“ (RP 199: 5). Ähnlich mahnt auch der Autor des Schwerpunktbeitrags im badischen Landesjugendamtsperiodikum Infoservice im Jahr 1999, Kinder- und Jugendhilfe solle sich trotz ihrer generellen „Anwaltsfunktion“ nicht in „fachmethodischer Hinsicht anwaltlich gerieren“ (Ba 199: 5,2). Denn damit gebe sie eine Orientierung am Prinzip der Hilfe zur Selbsthilfe auf und konzentriere sich auf ein „Herausboxen“ der Jugendlichen. Es helfe Jugendlichen aber nichts, so der Autor weiter, wenn ihre Taten verharmlost und sie selbst (auch dadurch) nur begrenzt ernst genommen werden. Jugendliche sollen lernen, Verantwortung für ihr eigenes Tun zu übernehmen (Ba 199: 5,2). Das aber setze ,ggf. auch Konfrontation voraus“. Es gehe darum, schreibt der Autor bereits einige Zeilen vorher, „mit allen Mitteln den Zugang zu gefährdeten Jugendlichen zu bahnen, d.h. authentisch und beherzt, ggf. auch mit Härte, aber fair" (Ba 199: 5,1). Ein solcher grundlegender Perspektivwechsel hin zu aktierungspädagogischen Strategien wird damit begründet, dass die heutige Welt für die Jugendlichen eine immer größere Unsicherheit bedeute. Auf der Thematisierungslinie der neuen Ungewissheit wird daher das Lernen eines Umgangs mit Unsicherheit als konstitutives Prinzip der Kinder- und Jugendhilfe gefordert: „Unsere Kinder werden vor Aufgaben gestellt sein, die schnellen Veränderungsprozessen unterliegen und so umfassend neu sein werden, dass sie für uns heute noch nicht einschätzbar sind. [...] Angesichts der Unvorhersehbarkeit der gesellschaftlichen Entwicklung kann das, was gelernt werden soll, nicht nur aus den aktuellen gesellschaftlichen Bedürfnissen abgeleitet und auf kognitive Leistungsfähigkeit, auf den Erwerb von Fähigkeiten und Fertigkeiten begrenzt werden. Es muss daneben um Bildungsprozesse gehen, um die Entwicklung der 
Gesamtpersönlichkeit, einer Persönlichkeit, die mit Selbstvertrauen und humaner Kompetenz die Gewissheit in sich trägt, auch neu offene Situationen bewältigen und gestalten zu können“, so schildert eine Mitarbeiterin des Allgemeinen Sozialen Dienstes (ASD) in Bremen in den vom dortigen Landesjugendamt publizierten SpielRäumen die neue Anforderungsstruktur, in die sie Kinder im Vorschulalter hinsichtlich ihrer Bildungsprozesse zu Beginn des 21. Jahrhunderts gestellt sieht (HB 300: 13,2). Diese Äußerung steht stellvertretend für häufig formulierte Einschätzungen, mit denen immer wieder betont wird, dass heutige Gesellschaften durch ein sehr hohes Tempo sozialen Wandels bestimmt seien (vgl. Rl 399: 4,1; Rl 399: 13,1). Im Bericht über eine Anhörung des rheinland-pfälzischen Landesjugendhilfeausschusses ist analog dazu davon die Rede, dass der „gesellschaftliche 'Ernstcharakter' die Kinder in vielerlei Hinsicht erreicht (habe)“" (RP 199: 8). Die Hamburger Jugendamtsleiterin sieht den sozialen Wandel gar als den entscheidenden Punkt an, an dem sich Kinder- und Jugendhilfe ausrichten müsse: „Anknüpfungspunkte sind Veränderungen der Lebenslagen von Kindern, Jugendlichen und ihren Familien, mit denen neue Anforderungen auf die Jugendhilfe zukommen" (HH 100: 2,1). Der rasante soziale Wandel führe dazu, dass sich Grade an Ungewissheit in der menschlichen Lebensführung stetig erhöhten. Kinder und Jugendliche sähen sich zunehmend der Anforderung gegenüber gestellt, Umgangsweisen auszubilden, die für solche Ungewissheitssituationen angemessen sind. Die nachwachsende Generation habe „Lebenskompetenzen zu erlernen ohne bereits abschätzen zu können, in welcher konkreten Lebenssituation diese Anwendung finden werden", wie das Bundesjugendkuratorium in diesem Zusammenhang formuliert (vgl. Münchmeier et al. 2002). Die „Halbwertzeit von als fest und sicher geglaubten Wissensbeständen“, so ist auch im 11. Kinder- und Jugendbericht zu lesen, nehme mehr und mehr ab (Bundestags-Drucksache 14/8181: 156).

Besonders bemerkenswert ist nun an den aktivierungspädagogischen Strategien im Feld der Kinder- und Jugendhilfe, dass diese Orientierung an den als konstitutiv ungewiss beschriebenen sozialen Zusammenhängen zugleich einhergeht mit einer Zunahme von Forderungen nach Verfahrensstandardisierung. Zum einen werden die Verhaltenscodices der bürgerlichen Mehrheitsgesellschaft weiter fixiert. Während allerdings im Rahmen wohlfahrtsstaatlicher Arrangements der nationalstaatliche Rahmen den Bezugspunkt von Verhaltensnormalisierungen darstellte, wird dieser nun kleinräumig und milieuspezifisch differenziert (Nahraumorientierung). Bevölkerungsstatistische Bestimmungen universeller Normalität werden zu gruppenbezogenen Bestimmungsverfahren transformiert und verfeinert (Sozialkartografie). Relevant für die Legitimierung sozialpädagogischer Interventionsstrategien ist nicht mehr primär der Armutsgrad einer Person in Relation zur Gesamtbevölkerung, sondern ihre Verhaltensweise mit Bezug auf die jeweilige nahräumliche Umgebung, das heißt eine bestimmte Bevölkerungsgruppe (,Unterschicht“) oder ein spezifisches Wohnareal („,benachteiligter Stadtteil“). Für welche dieser Räume Kinder- und Jugendhilfe zuständig ist, soll dann anhand „bestimmter Signale“ im kindlichen Verhalten (HB 299: 10,1), der Identifizierung „einzelner Kinder, die mehrfach durch deviantes Handeln auffallen“ (Sn 299: 18) oder der „Erforschung des Quartiers“ (HB 299: 1,3) festgestellt werden. Für die damit als normalisierungsbedürftig erfassten (individuellen wie kollektiven) Subjekte werden entsprechende 
nahraumorientierte Interventionsstrategien entwickelt und durchgeführt. Sozialstaatliches Regieren im nationalstaatlichen Rahmen scheint riskant geworden: Daher soll nun nahräumlich regiert werden.

Mit dieser skizzierten Zusammenschau dominierender Thematisierungslinien in den Feldern der bundesdeutschen Kinder- und Jugendhilfe lässt sich bereits deutlich deren aktivierungspolitische Re-Programmierung markieren. Während im bisherigen wohlfahrtsstaatlichen Arrangement das unterstützungsbedürftige Kind oder der unterstützungsbedürftige Jugendliche den Fokus sozialpädagogischer Maßnahmen darstellte, wird nun das noch nicht zur rational-kalkulativen Eigenverantwortung aktivierte oder zu aktivierende Kind bzw. der entsprechende Jugendliche als Teil einer kleinräumigen Gruppe benachteiligter Bevölkerungsmitglieder. zu deren Adressat erklärt. ${ }^{6}$ Diese gelte es zu identifizieren, um ihnen möglichst frühzeitig und mit aller Deutlichkeit ihre subjektive Lebensgestaltungsverantwortung zu verdeutlichen.

\section{Nach dem Ende sozialer Integrationsprogramme: Soziale Arbeit als gefragte aktivierungspolitische Partnerin}

Aktivierungspolitische Strategien sind inzwischen $\mathrm{zu}$ einem zentralen Rationalitätsmuster der Kinder- und Jugendhilfe geworden. Wenn diese Behauptung überzeugen kann - und die skizzierte dipositivanalytische Rekonstruktion aus den Feldern der bundesrepublikanischen Kinder- und Jugendhilfe legt dies nahe - so hat dies fundamentale Konsequenzen für Sozialpolitik und Sozialpolitikforschung.

Erstens sind aktivierungspolitische Strategien in ihrer aktivierungspädagogischen Ausprägung gerade auch in anderen Feldern als der Arbeitsmarktpolitik und der damit verbundenen Berufshilfe systematisch in den Blick zu nehmen. Nur so kann das Ausmaß der Wirkmächtigkeit der aktuellen politischen Neujustierungen des Sozialen erfasst werden. Zweitens sind aktivierungspädagogische Strategien als Teil einer grundlegenden neo-sozialen Transformation des bisherigen wohlfahrtsstaatlichen Arrangements zu rekonstruieren und zu bewerten. Und drittens ist zu realisieren, in welcher Weise bisher scheinbar emanzipativ oder reformerisch gedachte Redeweisen, wie die Prävention, die Stadtteilorientierung, aber auch die subjektive Autonomie oder die Eigenverantwortung neu konnotiert werden. Die mit diesen Begriffen selbstverständlich verbundenen Strategien der sozialpolitischen Erweiterung von Handlungsoptionen können so nicht mehr vorausgesetzt werden, sondern müssen wieder neu politisch erkämpft werden.

6 Die hier skizzierte dispositivanalytische Rekonstruktion stellt weder eine ideologiekritische Vorgehensweise dar, die auf ein „falsches Bewusstsein“ der Sprecher/innen hinweisen will, noch eine sprachanalytische Rekonstruktion, die auf tiefenhermeneutische Dimensionen einzelner Sprechakte aufmerksam machen möchte. Vielmehr werden dispositivanalytisch machtförmig wirksame und diskursiv artikulierte Regelmäßigkeiten des Sicht- und Sagbaren aufgezeigt, das heißt die historisch zu einem bestimmten Zeitpunkt wirksamen Prägungsmuster dechiffriert. Diese Prägungsmuster werden in der vorliegenden dispositivanalytischen Skizze als Thematisierungslinien bezeichnet (vgl. Kessl 2005). 
Welche Konsequenzen damit für die Auseinandersetzungen um Soziale Arbeit verbunden sind, soll abschließend angedeutet werden: Soziale Arbeit ist innerhalb wohlfahrtsstaatlicher Arrangements des Sozialen Teil des institutionalisierten Versprechens sozialer Integration. Soziale Ordnung wird hierbei als gesamtgesellschaftliche Aufgabe verstanden und staatliche Instanzen mit ihrer Aufrechterhaltung beauftragt. Sozialpädagogische Fachkräfte sind gleichzeitig alläglich damit konfrontiert, dass die Lebenslagen von Angebotsnutzern durch - teilweise immense - soziale Teilhabebeschränkungen gekennzeichnet sind (soziale Probleme). Klaus Mollenhauer (1993[1964]: 21) schien daher „die Gesellschaft im Sozialpädagogen einen ihrer heftigsten Kritiker“ zu erzeugen: „Durch die immer wieder neu auftretenden Schäden gibt sie der Kritik immer neue Nahrung“.

Mollenhauers Formel liest sich vor dem Hintergrund eines wohlfahrtsstaatlichen Programms (teil)kollektiver Risikokalkulation sehr einsichtig: Staatliche Instanzen werden mit der Aufrechterhaltung sozialer Ordnung beauftragt und zugleich erfahren die in diesen Feldern sozialpädagogisch Tätigen permanent, dass diese Ordnung nur unzureichend realisiert werden kann. Soziale Arbeit stellt insofern eine institutionelle Form gesellschaftlicher Selbsterinnerung an die Integrationsvereinbarung dar. Darüber herrscht(e) weitgehend Einigkeit. Gerät allerdings die wohlfahrtsstaatliche Vereinbarung selbst unter politischen Legitimationsdruck, wie dies aktuell unter dem Paradigma der Aktivierungspolitik zu beobachten ist, wird nicht nur einer derartigen gesellschaftskritischen Positionierung Sozialer Arbeit der Boden entzogen, sondern der Sozialen Arbeit im Sinne einer gesellschaftlichen Integrationsinstanz insgesamt. Denn thematisierbar sind Formen sozialer Desintegration nur solange die Übereinkunft zur kollektiven Risikokalkulation gültig bleibt (vgl. Castel 2005). Und eben diese wird von aktivierungspolitischen Programmstrategen zur Disposition gestellt. Bei unterschiedlicher Gewichtung neo-liberaler und neo-konservativer Anteile ist ihren Forderungen gemeinsam, dass eine aktivierende Sozialpolitik an Stelle der bisherigen versorgenden und insofern aktiven Sozialpolitik gerückt werden müsse. Als Ziel staatlicher Interventionsstrategien solle eine individuelle die bisherige (teil)kollektive Risikokalkulation ablösen. Das soziale Risiko wird zum privaten umdefiniert.

Soziale Arbeit ist gefragte Partnerin bei der Gestaltung dieser aktivierungspolitischen Transformationsprozesse. Denn die Pädagogisierung sozialpolitischer Denkweisen macht sie erforderlich, die Türwächter in die versprochene befreite Welt, in welcher die Nutzer ihre autonome Selbstführung realisieren sollen. Nur wurden viele der nun zu programmatischen Ehren kommenden sozialpädagogischen Selbstführungsprogramme in den ersten zwei Dritteln des 20. Jahrhunderts eben vor dem Hintergrund wohlfahrtsstaatlicher Arrangements des Sozialen, das heißt in einem grundlegend anderen Kontext, ausgearbeitet. Wenn die Sachverständigenkommission des 11. Kinder- und Jugendberichts Anfang des 21. Jahrhunderts in programmatischer Analogie konstatiert, dass ,,[d]er Aufbau einer personalen Identität ebenso wie die Übernahme gesellschaftlich anerkannter Vorgaben, Erwartungen und Zwänge, [...] für viele Jugendliche wesentlich als Selbstsozialisation [erfolgt] und [...] damit zunehmend durch die Jugendlichen selbst gesteuert [wird]“ (Bundestags-Drucksache 14/8181: 128), dann bekommt eine solche Selbstführungsforderung im Kontext aktivierungspolitischer Trans- 
formationsprozesse einen bitteren Beigeschmack. Denn der Primat der Selbstführung geht inzwischen als Programm subjektiver Lebensgestaltungsverantwortung eine verheerende Symbiose mit einer wachsenden öffentlichen Verantwortungslosigkeit ein. Die geforderte subjektive Selbstführung, die Übernahme von Lebensgestaltungsverantwortung, setzt voraus, dass Akteure Einfluss nehmen können auf eben diese Gestaltung ihrer Handlungsvollzüge. Verfügungsmöglichkeiten über Handlungsressourcen geraten aus dem Blick, wenn Kinder- und Jugendhilfe im Konzert mit anderen Protagonisten aktivierungspolitischer Strategien zunehmend Verhaltenstrainings statt Verhältnisproblematisierung betreibt. Die aktuell dynamisierten Strategien einer „subjektiven Verantwortlichmachung“, wie sie am dargestellen Material illustriert wurden, entziehen einer gegenseitigen Verantwortungsübernahme, das heißt kollektiven Solidaritätsstrukturen, systematisch den Boden. Der Einzelne soll sich in Konkurrenz und Wettbewerb mit den anderen begreifen, sein eigenes Handeln nach „,subjektiven“ Effizienz- und Effektivitätskriterien kalkulieren, wie die thüringische Schulklasse im Rahmen des Nichtraucherwettbewerbs. Gefragt sein soll nicht mehr eine grundsätzliche strukturelle Solidarität sozialer Sicherungsstrukturen, denn diese scheint den aktivierungspolitischen Protagonisten ineffektiv und hinderlich. Wer sich der subjektiven „Verantwortlichmachung" verweigert, zeige dadurch, so die aktivierungspolitische Unterstellung, dass er auf öffentliche Unterstützung verzichten wolle. Wenn vor dem Hintergrund einer fortgeschritten liberalen Gestalt des Sozialen soziale Teilhabebeschränkungen oder mangelnde Bildungsteilhabe die Konsequenzen einer solchen „subjektiven Entscheidung“ sind, ist das für „,die Gesellschaft“ kein relevantes moralisches Problem mehr. Demgemäß kann der ehemalige nordrhein-westfälische Ministerpräsident - der inzwischen zum Bundesminister für Finanzen avancierte formulieren: „Soziale Gerechtigkeit muss für uns heißen, eine Politik für jene zu machen, die etwas für die Zukunft unseres Landes tun. [...] Um sie - und nur um sie - muss sich Politik kümmern“ (Die Zeit vom 17.12.2003).

Solchen aktivierungspolitischen Plädoyers sollte eine kritisch-reflexive Soziale Arbeit und Kinder- und Jugendhilfe als Teil einer kritischen Sozialpolitik entgegenwirken. Wohlfahrtsstaatliche Arrangements sind dazu allerdings nicht blind zu verteidigen. Vielmehr sollte dafür gestritten werden, was der Wohlfahrtsstaat als kollektives Arrangement individueller Freiheitsrechte und kollektiver Gleichheitsrechte sein könnte.

\section{Literaturverzeichnis}

Bandemer, Stephan; Hilbert, Josef (1998): „Vom expandierenden zum aktivierenden Staat", in: Bandemer, Stephan; Blanke, Bernhard; Nullmeier, Frank; Wewer, Göttrik (Hg.): Handbuch zur Verwaltungsreform. Opladen: Leske und Budrich, 25-32.

Bundestags-Drucksache 14/8181 (2002): Bericht über die Lebenssituation junger Menschen und die Leistungen der Kinder- und Jugendhilfe in Deutschland (11. Kinderund Jugendbericht). Berlin: Bundesministerium für Familie, Senioren, Frauen und Jugend. 
Castel, Robert (2005): Die Stärkung des Sozialen: Leben im neuen Wohlfahrtsstaat. Hamburg: Hamburger Edition.

Dahme, Heinz-Jürgen; Otto, Hans-Uwe; Trube, Achim; Wohlfahrt, Norbert (Hg.) (2003): Soziale Arbeit für den aktivierenden Staat. Opladen: Leske und Budrich.

Dahme, Heinz-Jürgen; Wohlfahrt, Norbert (2005): Aktivierende Soziale Arbeit: Theorie Handlungsfelder - Praxis. Baltmannsweiler: Schneider.

DiFabio, Udo (2005): Die Kultur der Freiheit. München: Beck.

Dingeldey, Irene (2003): „Politikfeldübergreifende Koordination als neue Steuerungsform im aktivierenden Sozialstaat? Eine Analyse der Employability Politik in Dänemark, Deutschland und Großbritannien am Beispiel der Beschäftigungsfähigkeit von Müttern“, in: Österreichische Zeitschrift für Politikwissenschaft 37: 97-107.

Dingeldey, Irene (2005): Wandel von Governance im Sozialstaat. Zur Implementation aktivierender Arbeitsmarktpolitik in Deutschland, Dänemark und Großbritannien. SFB597 „Staatlichkeit im Wandel“/,Transformations of the State“. TranState Working Papers No. 12. Bremen.

Ehrke, Michael (1999): Der Dritte Weg und die europäische Sozialdemokratie. Ein politisches Programm für die Informationsgesellschaft? Bonn: Friedrich-Ebert-Stiftung.

Eick, Volker; Sambale, Jens (2005): Sozialer Wohnungsbau, Arbeitsmarkt(re)integration und der neoliberale Wohlfahrtsstaat in der Bundesrepublik und Nordamerika. Berlin: Freie Universität.

Esch, Karin; Hilbert, Josef; Stöbe-Blossey, Sybille (2001): „Der aktivierende Staat - Konzept, Potentiale und Entwicklungstrends am Beispiel der Jugendhilfe“, in: Heinze, Rolf G.; Olk, Thomas (Hg.) (2001): Bürgerengagement in Deutschland: Bestandsaufnahmen und Perspektiven. Opladen: Leske und Budrich, 519-547.

Evers, Adalbert (2000): „Aktivierender Staat - eine Agenda und ihre möglichen Bedeutungen“, in: Mezger, Erika; West, Klaus-W. (Hg.): Aktivierender Sozialstaat und politisches Handeln. Marburg: Schüren, 13-39.

Freyberg, Thomas (2003): „Fördern und Fordern, Teil 1: Der Angriff auf den deutsche Sozialstaatskonsens“, in: Widersprüche, 23 (Heft 88): 117-134.

Gerdes, Jürgen (2006, i.E.): „Der 'Dritte Weg' als wissentliche Kolonialisierung der Lebenswelt. Zur Aktualität der Kolonialisierungsthese angesichts der programmatisch-ideologischen Modernisierung im Kontext der Idee der Wissensgesellschaft", in: Bittlingmayer, Uwe; Bauer, Uwe (Hg.): Die Wissensgesellschaft. Mythos, Ideologie oder Realität?. Wiesbaden: VS.

Gilbert, Neil; Gilbert, Barbara (1989): The Enabling State. Modern Welfare Capitalism in America. New York: Oxford University Press.

Gillen, Gabriel (2004): Hartz IV: eine Abrechnung. Hamburg: Rowohlt.

Hayek, Friedrich (1960/1991): Verfassung der Freiheit. Tübingen: Mohr.

Hasenclever, Christa (1978): Jugendhilfe und Jugendgesetzgebung seit 1900. Göttingen: Vandenhoeck und Ruprecht.

Hombach, Bodo (1998): Aufbruch: die Politik der neuen Mitte. München: Econ.

Kaufmann, Franz-Xaver (2002): Sozialpolitik und Sozialstaat: Soziologische Analysen. Opladen: Leske und Budrich.

Kessl, Fabian (2005): Der Gebrauch der eigenen Kräfte: eine Gouvernementalität Sozialer Arbeit. Weinheim und München: Juventa. 
Kessl, Fabian (2006): „Individualität - Dein Risiko! Soziale Arbeit auf dem Weg zur neosozialen Risikokalkulatorin“, in: Dollinger, Bernd (Hg.) (2006): Individualität als Risiko? Soziale Pädagogik als Moderisierungsmanagement. Münster: Lit.

Kessl, Fabian; Otto, Hans-Uwe (2003): „Aktivierende Soziale Arbeit. Anmerkungen zur neosozialen Programmierung Sozialer Arbeit“, in: Dahme, Heinz-Jürgen; Otto, Hans-Uwe; Wohlfahrt, Norbert; Trube, Achim (Hg.) (2003): Soziale Arbeit für den aktivierenden Staat. Opladen: Leske und Budrich, 57-73.

Lessenich, Stephan (2003): „Soziale Subjektivität: die neue Regierung der Gesellschaft“, in: Mittelweg 36 12/4: 80-93.

Lessenich, Stephan; Möhring-Hesse, Matthias (2005): „Ein neues Leitbild für den Sozialstaat: eine Expertise im Auftrag der Otto-Brenner-Stiftung (Auszüge)“, in: Dies.; Nahles, Andrea; Peters, Jürgen; Stolterfoht, Barbara u.a. (2005): Den Sozialstaat neu denken. Hamburg: VSA, 87-156.

Maaser, Wolfgang (2006): „Der Aktivierungsdiskurs der Verantwortung“, in: Neue Praxis 36/1: 37-52.

Mahnkopf, Birgit (2000): „Formel 1 der Sozialdemokratie. Zur Neuinterpretation der sozialen Frage im globalen Kapitalismus“, in: Prokla 30: 489-525.

Marston, Greg; McDonald, Catherine (2006): Analysing Social Policy A Governmental Approach. Cheltenham: Edward Elgar.

Mollenhauer, Klaus (1993[1964]): Einführung in die Sozialpädagogik: Probleme und Begriffe der Jugendhilfe. Weinheim und Basel: Beck.

Münchmeier, Richard; Otto, Hans-Uwe; Rabe-Kleberg, Ursula (Hg.) (2002): Jugendhilfe und Lebenskompetenz: Kinder- und Jugendhilfe vor neuen Aufgaben. Opladen: Leske und Budrich.

Nolte, Paul (2006): Riskante Moderne. Die Deutschen und der neue Kapitalismus. München: Beck.

Olk, Thomas (2000): „Der ,aktivierende Staat‘. Perspektiven einer lebenslagenbezogenen Sozialpolitik für Kinder, Jugendliche, Frauen und älter Menschen“, in: Siegfried Müller; Heinz Sünker; Thomas Olk; Karin Böllert (Hg.) (2000): Soziale Arbeit. Gesellschaftliche Bedingungen und professionelle Perspektiven. Neuwied: Kriftel, 99-118.

Oschmiansky, Frank: „Faule Arbeitslose? Zur Debatte über Arbeitsunwilligkeit und Leistungsmissbrauch“, Aus Politik und Zeitgeschichte B06-07/2003: 10-16.

Petersen, Käthe (1976): Selbsthilfe und ihre Aktivierung durch die Soziale Arbeit. Gesamtbericht über den 68. Deutschen Fürsorgetag 1976 in Dortmund, 10.-12. November 1976. Frankfurt a.M.: Schriften des Deutschen Vereins für öffentliche und private Fürsorge.

Richter, Martina: „Zur (Neu)Ordnung des Familialen“, Widersprüche 24 (Heft 92): 7-16.

Schaarschuch, Andreas (2003): „Am langen Arm: Formwandel des Staates, Staatstheorie und Soziale Arbeit im entwickelten Kapitalismus“, in: Hans-Günther Homfeldt; Jürgen Schulze-Krüdener (Hg.) (2003): Handlungsfelder der Sozialen Arbeit, Band 3. Stuttgart: Kohlhammer, 36-65.

Schmidt-Semisch, Henning (2002): Kriminalität als Risiko: Schadenmanagement zwischen Strafrecht und Versicherung. München: Gerling Akademie. 
Sinn, Hans-Werner; Holzner, Christian; Meister, Wolfgang; Ochel, Wolfgang; Werding, Martin (2002): Aktivierende Sozialhilfe: ein Weg zu mehr Beschäftigung und Wachstum. Ifo-Schnelldienst, Heft 9. München.

Streeck, Wolfgang; Heinze, Rolf G. (2000): „Wende am Arbeitsmarkt durch Erneuerung des ,deutschen Modells“", Sozialwissenschaftliche Informationen 29/1: 28-38.

Sturzenhecker, Benedikt (2003): „Aktivierende Jugendarbeit?“, in: Heinz-Jürgen Dahme; Hans-Uwe Otto; Achim Trube; Norbert Wohlfahrt (Hg.) (2003): Soziale Arbeit für den aktivierenden Staat. Opladen: Leske und Budrich, 381-390.

Walther, Andreas (2003) „Varianten zwischen Erpressung und Empowerment. Für eine Erweiterung des Diskurses zum aktivierenden Statt im internationalen Vergleich“, in: Neue Praxis 33: 288-305.

Widerspruch (2004): Marktregime und Subjekt im Neoliberalismus. 24. Jg., Heft 46.

Widersprüche (2005): Klassengesellschaft reloaded. Zur Politik der „neuen Unterschicht“. 25. Jg., Heft 98.

Winkler, Michael (1988): Eine Theorie der Sozialpädagogik: über Erziehung als Rekonstruktion der Subjektivität. Stuttgart: Klett Cotta.

Winkler, Michael (2006): Kritik der Pädagogik. Der Sinn der Erziehung. Stuttgart: Kolhammer 2006.

Wölfle; Tobias; Schöller, Oliver (2004): „Soziale Disziplinierung im flexiblen Kapitalismus", in: Prokla 34: 339-356.

Ziegler, Holger: Jugendhilfe als Prävention : die Refiguration sozialer Hilfe und Herrschaft in fortgeschritten liberalen Gesellschaftsformationen. Dissertationsschrift, Universität Bielefeld. Download unter: http://bieson.ub.unibielefeld.de/volltexte/2004/533/ (Stand: 18. Februar 2006). 